\title{
Symptomatic spinal epidural lipomatosis after combined hormonal and steroidal palliative therapy of prostate cancer
}

\author{
Amir Awwad $\mathbb{B}^{1,2} \cdot$ Renan E. Ibrahem Adam ${ }^{3} \cdot$ Chandni Patel ${ }^{4} \cdot$ James D. Thomas ${ }^{3}$
}

Received: 19 February 2018 / Revised: 30 June 2018 / Accepted: 30 June 2018

(c) International Spinal Cord Society 2018

\begin{abstract}
Introduction Spinal epidural lipomatosis (SLE) is an abnormal accumulation of unencapsulated fat. The association of chronic steroid therapy is described as well as obesity and Cushing's syndrome. SLE has a range of neurological presentations such as back pain, myelopathy, and cauda equina syndrome. Surgical decompression is known to improve symptomatic cases and prevent further deterioration.

Case presentation An elderly male patient with background history of high-risk metastatic prostate cancer and long-term treatment with steroids and hormonal therapy presented with acute on chronic back pain and neurological symptoms. Spinal cord compression was excluded on the subsequent MRI. However, a spinal epidural lipomatosis causing significant central canal stenosis and compression of the cauda equine was revealed.

Discussion With the increasing role of MRI in assessing back pain, asymptomatic spinal epidural lipomatosis has been frequently noticed. However, symptomatic presentation after long term combined treatment of steroids and hormonal therapy has not been reported in the literature.
\end{abstract}

\section{Introduction}

Spinal epidural lipomatosis (SLE) is a rare cause of back pain and neurological symptoms. SLE is characterized by hyperplasia of normal epidural fat. This could potentially enlarge and compress spinal cord and terminal nerve roots resulting in a neurological deficit. Standard MRI sequences can reliably diagnose SLE by depicting the abundant fat accumulation as high T1, T2-weighted signals with its corresponding signal nulling on fat suppressing imaging techniques. SLE should be considered in the differential

James D. Thomas

james.thomas3@nuh.nhs.uk

1 NIHR Nottingham Biomedical Research Centre, Sir Peter Mansfield Imaging Centre (SPMIC), University of Nottingham, Nottingham NG72UH, UK

2 Radiology Department, Royal Papworth Hospital NHS Foundation Trust, Cambridge CB23 3RE, UK

3 Radiology Department, Queen's Medical Centre, Nottingham University Hospitals NHS Trust, Derby Road, Nottingham NG7 2UH, UK

4 Urology Department, Nottingham Urology Centre, City Hospital, Nottingham NG5 1PB, UK diagnosis when assessing patients with resistant prostate cancer on long-term steroids and hormonal treatment. Asymptomatic cases have been previously reported, however to our knowledge there has been no previous case report of symptomatic enlargement of the epidural fat following combined treatment with steroids and hormonal therapy in the literature.

\section{Case presentation}

A 75-year-old gentleman was diagnosed with oligometastatic high-risk prostate carcinoma (Gleason 8) in 2010. He was initially treated for 2 years with Goserelin (Zoladex ${ }^{\circ}$ ), a gonadotrophin releasing hormone superagonist. This has a suppressive effect on sex hormone production (testosterone and estrogen); used particularly for breast and prostate cancer hormonal therapy. In 2012, Bicalutamide (Bicalutamide ${ }^{\circ}$ ), a totally synthetic androgen blocker (non-steroidal), was introduced due to bone metastases. Urological assessment confirmed that there were objective symptoms of poor urinary stream and nocturia. Therefore, the patient underwent a transurethral resection of prostate after which he required a long-term urinary catheter. Subsequently, surveillance over 12 months 


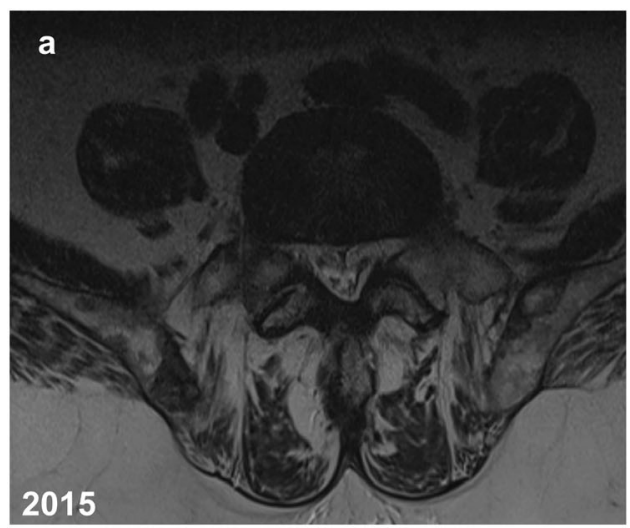

Fig. 1 a, b A 75-year-old man with advanced metastatic prostate cancer. Axial T2-weighted MR images of the lower lumbar spine a 2015 (1.5 Tesla, TR $4671 \mathrm{~ms}$, TE $92 \mathrm{~ms}$, slice thickness $4 \mathrm{~mm}$ ) and b 2017 (1.5 Tesla, TR $2260 \mathrm{~ms}$, TE $110.76 \mathrm{~ms}$, slice thickness $4 \mathrm{~mm}$ ).

showed that assays of prostate-specific antigen (PSA) began to rise and the Bicalutamide treatment after 2 years was replaced by regular dexamethasone (daily $0.5 \mathrm{mg}$ ).

In 2014, it was decided to start the patient on Abiraterone (Zytiga). This is a new type of hormonal therapy licensed for advanced prostate cancer with refractory response to other hormone therapy regimens, evidently for its new advantages of prolonged survival and symptoms control. However, after 6 months, this was not very tolerable by the patient whose subsequent treatment as a result only consisted of Docetaxel (Taxotere ${ }^{\oplus}$ ) in combination with low dose Prednisolone.

In 2015, the Docetaxel (Taxotere ${ }^{\circ}$ ) was stopped after four cycles along with the Prednisolone due to recurrent chest infection with no significant clinical or biochemical response. Dexamethasone was then re-started even as the PSA continued to increase. Restaging CT and bone scans showed progression mainly with dissemination of bony deposits, but no soft tissue or visceral metastatic sites detected elsewhere. As a result, he was commenced on Radium $^{223}$ and tolerated only four injections but with a good response to therapy. The last Radium ${ }^{223}$ injection was in summer 2015.

Early 2017, the patient presented with an acute on chronic back pain and reduced mobility for a few days with ongoing history of stool incontinence. He had previously described mild symptoms of chronic back pain for over a year. The neurological examination of the lower limbs revealed mild bilateral reduction motor strength (4 of 5), presumably attributed to pain, with grossly intact sensory functions. Thus, an intact single-neurological level (SNL) estimated at T12 with mild anal tone reduction (incomplete sacral sparing) suggested an overall spinal cord injury (SCI) grade D [1]. The rest of the examination was unremarkable. His past medical history included ischemic heart disease,

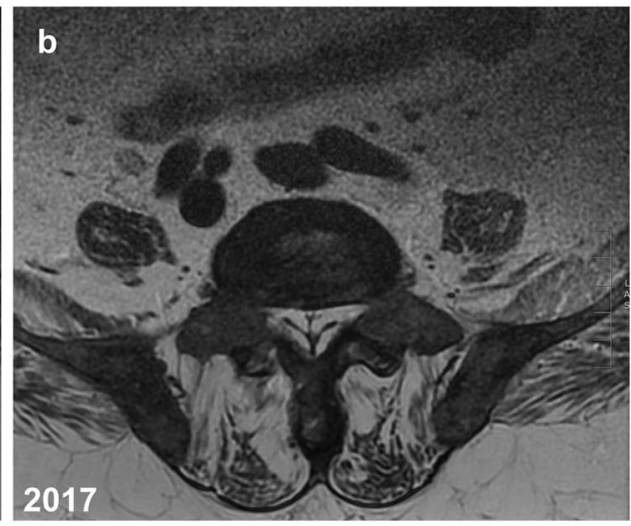

By comparison, images show increasing abundant epidural fat compressing the thecal sac with obvious tapering, especially b 2017 image demonstrating a "Y" sign indicative of high-grade thecal sac compression

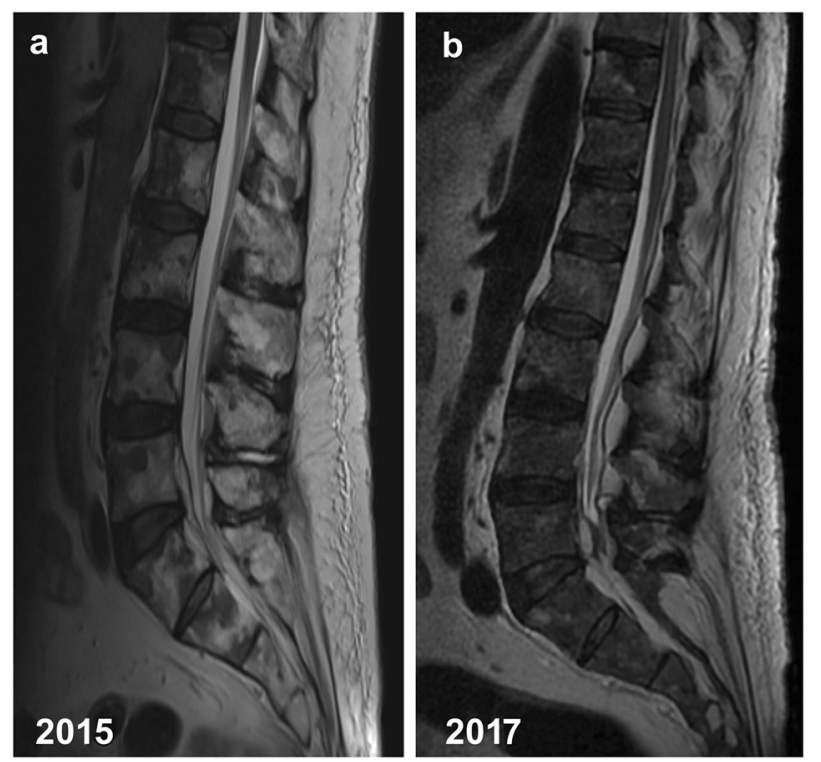

Fig. 2 a, b A 75-year-old man with advanced metastatic prostate cancer. Sagittal T2-weighted MR images of the lumbar spine a 2015 (1.5 Tesla, TR $3490 \mathrm{~ms}$, TE $88 \mathrm{~ms}$, slice thickness $4 \mathrm{~mm}$ ) and b 2017 (1.5 Tesla, TR $3720 \mathrm{~ms}$, TE $112.92 \mathrm{~ms}$, slice thickness $4 \mathrm{~mm}$ ). The images show increased interval accumulation of epidural fat especially in the lower lumbar and sacral region

hypertension, emphysema, and rheumatoid arthritis. For a summary of chronological treatment history, please see Table 1.

The presentation triggered an urgent spinal MRI request by the medical receiving unit which was performed on the same day to rule out metastatic cord compression. The MRI scan covering the whole spine revealed unusually abundant epidural fat throughout the thoracic and lumbar region narrowing the spinal canal and displacing the thecal sac and the thoracic cord anteriorly (Figs. 1 and 2). The caudal thecal sac was found to be markedly constricted by the 
Table. 1 In chronological order, the table lists a summary of treatment events received by the patient since initial diagnosis until the case presentation in 2017

\begin{tabular}{ll}
\hline Date & Treatment history \\
\hline January 2010 & Diagnosed with oligo-metastatic prostate CA, started on Zoldadex (for 2 years) \\
March 2012 & Bicalutamide introduced due to bone mets (for 2 years) \\
January 2013 & Urological symptoms, Surgical Prostate Resection \\
February 2014 & PSA rising, Bicalutamide replaced by regular Dexamethasone \\
April 2014 & Start Abiraterone (only for 6 months due to patient's intolerability) \\
September 2014 & Stopped Abiraterone and replaced by Docetaxel and low dose prednisolone \\
January 2015 & Stopped Docetaxel \& Prednisolone stopped due to complications \\
January 2015 & Only on Dexamethasone, PSA continued to rise \\
February 2015 & CT shows bone metastases only, then started Radium ${ }^{223}$ radiotherapy \\
July 2015 & Completed $4 \times$ Radium ${ }^{223}$ cycles, tolerated well with good response \\
January 2017 & Presented with acute on chronic lower back pain \\
\hline
\end{tabular}

In chronological order, the table lists a summary of treatment events received by the patient since initial diagnosis until the case presentation in 2017 epidural fat. Multiple bony metastases and low-grade pathological fractures were also noted, some of which were noted previously, and some were new. He was admitted under the care of the uro-oncological team, where he was treated conservatively with analgesia, red cell transfusion, and intravenous fluid therapy. Clinically he improved within a week and was discharged home. He subsequently died 3 months later.

\section{Discussion}

Spinal epidural lipomatosis describes a rare and unusual pathological overgrowth of the adipose tissue in the epidural space. This is likely due to hypertrophy of the dural adipose tissue, which can cause spinal canal narrowing and cord compression if severe. It is most commonly seen in patients receiving exogenous steroid therapy, but can also be found in those with endocrinopathies, endogenous corticosteroid overproduction, obesity, or idiopathic disease [2, 3]. Lee et al. has first described SEL in 1975, in a post renal transplantation patient [4]. Recently, Mattei et al. presented a case of metastatic prostate cancer with symptomatic SEL attributed to multimodal treatment with androgen receptor antagonist [5]. To our knowledge, there has been no fully published case report on the progressive and symptomatic enlargement of epidural fat following antiandrogen treatment.

The course of the condition is usually insidious and subtle. Symptoms vary and may start with vague back pain, which can progress to weakness and sensory loss in the lower extremities. The neurological symptoms depend upon the level of the spinal canal and neural structures (spinal cord, conus medullaris, or cauda equina) involved. Most commonly, if the thoracic levels (58-61\% of cases) are affected it could produce progressive myelopathy, with associated spinal cord compression producing generalized motor and sensory loss [3]. Lumbar involvement (39-42\% of cases) can produce radiculopathy caused by potential compression to specific nerve roots with localized symptomatology [6]. Bowel and bladder incontinence are rare. The developed condition is likely to affect the thoracic and the lumbar spinal regions in equal proportions [7].

MRI is the modality of choice with superior and selective capabilities to distinguish fat content within the imaged fields of view. Generally, T1-weighted images of adipose tissue will demonstrate high signal intensity; however, this can also be seen in water-filled areas in tissues. The widely used fat-suppression sequences (short tau inversion recovery, STIR) can then confirm the loss of such signal when due to pure fat content. In this particular case this helped to detect the abnormal and abundant extradural fat accumulation (Figs. 1 and 2). MRI typically shows a polygonal spiculated Y shaped or stellar morphological deformation of the dural sac in association with epidural fat overgrowth. It is thought that these characteristic spiculations correspond to the dural insertions of the meningo-vertebral ligaments while the depressions correspond to the accumulated epidural fat [7]. This configuration of deformed fat overgrowth could potentially cause spinal canal and/or trans-foraminal narrowing. Spinal epidural lipomatosis should be differentiated from compressing spinal lipomas which are usually focal and encapsulated.

Depending on the degree of neurological deficits, treatment options range from conservative measures to spinal surgical intervention [8]. Weaning off steroid therapy and weight loss are two of the conservative options. However, some patients receive steroid therapy for chronic conditions and may not tolerate treatment cessation. In absence of exogenous steroid therapy, evaluation for possible endogenous corticosteroid overproduction requires investigation [9]. Following that, surgical intervention should be 
considered. Decompressive laminectomy and excision of the adipose tissue is a successful surgical intervention carried out in many patients. The procedure involves removal of the lamina segment of the vertebrae at the level of the epidural lipomatosis, relieving the compression. It appears that a majority of these patients experience improvement or resolution of their neurological symptoms after surgical intervention [3]. In the case of idiopathic SEL, a recent surgical publication reviewed 14 reported cases in literature between 1988 and 2017 of complete recovery and improved myelopathy following spinal decompression, which were all thoracic (T1-T10) SEL cases [10].

With the increased use of combined steroids and hormonal therapy in metastatic resistant prostate cancer, it is very important to be aware of spinal epidural lipomatosis as a rare complication. It should be considered in the differential diagnosis when spinal cord compression has been excluded in patients presenting with chronic back pain and neurological symptoms.

Author contributions All listed authors have read, approved, and contributed sufficiently to take responsibility for the whole content of the manuscript following the criteria in ICJME guidelines for authorship rights and responsibilities.

\section{Compliance with ethical standards}

Informed consent An informed written consent for publication has been obtained from the patient.

Conflict of interest The authors declare that they have no conflict of interest.

\section{References}

1. Kirshblum SC, Burns SP, Biering-Sorensen F, Donovan W, Graves DE, Jha A, et al. International standards for neurological classification of spinal cord injury (revised 2011). J Spinal Cord Med. 2011;34(6):535-46.

2. Akiyama N, Takeuchi M, Shibagaki Y, Uchiyama S, Iwata M. [Two cases of spinal epidural lipomatosis]. Rinsho Shinkeigaku. 1999;39(6):634-8.

3. Fassett DR, Schmidt MH. Spinal epidural lipomatosis: a review of its causes and recommendations for treatment. Neurosurg Focus. 2004;16(4):E11.

4. Lee M, Lekias J, Gubbay SS, Hurst PE. Spinal cord compression by extradural fat after renal transplantation. Med J Aust. 1975;1 (7):201-3.

5. Mattei T, Goulart C, A Rehman A, Mendel E. Massive symptomatic epidural lipomatosis after multimodal therapy with androgen receptor antagonists for metastatic castration-resistant prostate cancer. In: AANS Conference Proceedings (Poster-ID 31000); American Association of Neurological Surgeons, Washington DC, USA, 2015.

6. Alvarez A, Induru R, Lagman R. Considering symptomatic spinal epidural lipomatosis in the differential diagnosis. Am $\mathrm{J}$ Hosp Palliat Med. 2012;30(6):617-9. https://doi.org/10.1177/ 1049909112457012.

7. Choi K-C, Kang B-U, Lee CD, Lee S-H. Rapid progression of spinal epidural lipomatosis. Eur Spine J. 2012;21(Suppl 4): S408-12.

8. Wells AJ, McDonald MJ, Sandler SJI, Vrodos NJ. Lumbosacral epidural lipomatosis causing rapid onset cauda equina syndrome. $\mathrm{J}$ Clin Neurosci. 2014;21(7):1262-3.

9. Praver M, Kennedy BC, Ellis JA, D'Amico R, Mandigo CE. Severity of presentation is associated with time to recovery in spinal epidural lipomatosis. J Clin Neurosci. 2015;22(8):1244-9.

10. Al-Yafeai R, Maghrabi Y, Malibary H, Baeesa S. Spinal cord compression secondary to idiopathic thoracic epidural lipomatosis in an adolescent: a case report and review of literature. Int J Surg Case Rep. 2017;37:225-9. 